MINOR CONTRIBUTION.

\title{
A New Method of Determining The Specific Heats of Liquids.
}

By Robert L. Litch.

$\mathrm{T}$

HE following methods have been used for determining the specific heats of liquids: (I). Method of cooling. (2). Method of mixtures. (3). ${ }^{1}$ Method of Thomson. (4). Electrical methods as proposed by Jamin ${ }^{2}$ and Anmary, Weber, Pfaundler, Dieterici. ${ }^{3}$

The errors to which these methods are liable are too well known to need comment. I propose in this paper to outline a new method which shall involve not only the virtues lying in the so-called law of Joule but also the principle which is the foundation of the "Waterman" Calorimeter for the method of mixtures," viz., the maintaining of the calorimeter cup at a constant temperature throughout the experiment. By this principle the errors due to the uncertainty as to what corrections must be made for "Water Equivalent" and "Radiation correction" are avoided.

\section{Theory of the Method.}

Witnin two vessels, arranged one above the other, the liquid whose specific heat is desired is placed. This particular arrangement enables the operator to easily transfer liquid from one vessel to the other. The assumption is made that the temperature of the two are different, that of the upper vessel being the lower. Though theory shows it is not necessary, yet practice has made it appear advisable to make the temperature of the cooler liquid as near $\circ^{\circ} \mathrm{C}$. as possible. The temperature of the other is that of the room.

In the lower vessel a coil of wire is also placed. A current of known strength is passed through the coil and the temperature of the liquid is, in consequence, raised. Enough liquid is dropped into the lower vessel from the upper to maintain the temperature of the liquid in the lower constant during the operation.

Then it is evident that

$$
H=\operatorname{sm}\left(T-T_{0}\right)
$$

1 Pogg. Ann. I42, pp. 337.

${ }^{2}$ Comptes Rendus, LXX., pp. 66r.

3 Wied. Ann. 33, pp. 417.

${ }^{4}$ Phys. Rev., Vol. IV., No. 2 I. 
where $H=$ heat developed

$s=$ specific heat

$m=$ mass of liquid dropped

$T-T_{0}=$ difference in temperature between that of the cooler liquid and the room.

If we substitute this value in the expression for Joule's Law, we have

$$
\text { (I) } s=\frac{r c^{2} t}{\operatorname{Jm}\left(T-T_{0}\right)}
$$

where $r, c, t$ and $J$ have the usual meaning.

If we take two such calorimeters containing different liquids and allow the same current to pass through them it is evident that

$$
\text { (II) } \frac{s_{1}}{s_{2}}=\frac{m_{2} r_{1}\left(T-T_{02}\right)}{m_{1} r_{2}\left(T-T_{01}\right)}
$$

where the subscripts refer to the two calorimeters.

\section{Apparatus.}

My apparatus is constructed in many respects on the lines of the "Waterman Calorimeter." The cooler and air-thermometer are direct copies of the corresponding parts in that apparatus. The test-tube, however, is made of very thin glass and not of silver. It passes through a cork and with it completely closes the bulb of an air-thermometer. Inside the tube is a coil of platinum wire whose ends pierce the sides of the tube and are soldered to large copper wires. These wires serve as "leads" and run up along the sides of the tube, entering the open air through the cork at the mouth of the bulb.

The other pieces of apparatus found necessary are: (I) Two thermometers, one graduated to $\frac{1}{20}$ degree, so arranged in the cooler that the liquid flows over its bulb just at the moment of passing from cooler to test tube, and another graduated to $\frac{1}{5}$ degree for measuring the room temperature; (2) a copper voltameter; (3) a D'Arsonval galvanometer for the measurement of resistance by the fall of potential method; (4) storage batteries; (5) miscellaneous apparatus, such as variable resistance-boxes, direct reading ammeter, keys, etc.

\section{Practice of the Method.}

The operations necessary are: (a) determination of current; (b) determination of the amount of liquid dropped; $(c)$ measurement of time ; $(d)$ measurement of resistance of coil ; $(e)$ observation of the temperatures of the liquid in the two vessels; $(f)$ substitution in the formula

$$
s=\frac{c^{2} r t}{J m\left(T-T_{0}\right)}
$$


In my experiments I have used an ordinary watch. This has been possible because by using small currents the experiment could be continued from five to ten minutes, and hence, the total number of seconds being large, an error of one second would introduce no perceptible change in the results. With a spring contact key, such as I have used, it is possible to turn the current on and off so near the second intended that the sum of the errors introduced by these two operations is undoubtedly much less than a second.

The measurement of resistance is the most important of all the operations. It is necessary to measure this during the experiment itself. It is not sufficient to know it at zero degrees and its temperature coefficient, because the exact temperature of the coil cannot be determined while the operation is going on. To regard it as being the same as the liquid is erroneous, for it is easy to see that, were it so, no flow of heat could take place from the coil to the liquid. To overcome this difficulty I have made use of the fall of potential method for this observation. This has the virtue of being easy of application and, for the small resistances I have used, of great accuracy. For my coil of known resistance, because of its slight change resistance due to a rise in temperature, large German-silver wire (No. I6, B. W. G.) was employed. This arrangement has been found very satisfactory.

\section{Results Obtained.}

Owing to various reasons I have been unable to apply my method to but one substance, viz., water. Even with this I have taken no great precaution that it should be absolutely pure and hence the results are given merely to show how the method works. Rowland's statement that the specific heat of water decreases between $4^{\circ}$ and $30^{\circ} \mathrm{C}$. is seen to be followed in these results :

\begin{tabular}{lc}
\multicolumn{1}{c}{$T$} & s \\
$18.8^{\circ}$ & .98075 \\
19.7 & .98064 \\
21.05 & .98035 \\
21.2 & .98035
\end{tabular}

These values lie between those given by Dieterici and Velten for $20^{\circ}$, the former obtaining .9893, the latter .9794. The method of mixtures has invariably given for this temperature values greater than unity.

\section{Second Method.}

The values of $J$ used in the experiments under the first method are those due to Rowland. They are probably the best so far obtained but notwithstanding this fact there is undoubtedly a great degree of inde- 
terminateness as to the correct value of this constant and hence it is best to eliminate it, if possible, from any method of experimentation.

A glance at equation (II) reveals the fact that we require no knowledge of (I) current, (2) time, (3) mechanical equivalent. Why these three quantities disappear from the equation appears in the following considerations. The two calorimeters necessary for the practice of this method have their coils joined in series and hence the same current flows through both. The consequence is that whatever fluctuation in the current occurs, the effect in one is as great as in the other. It follows, therefore, that at every instant the current through the first is equal to that through the second and hence the ratio of the two is vigorously unity. Similar reasoning will show why the other two quantities are eliminated.

The first method requires a knowledge of all three and in addition we are compelled to make use of the average current. Trouble arises here because when set into the formula it must be squared giving a value $\left[\mathrm{I} / t \sum i\right]^{2}$ whereas we desire $\mathrm{I} / t \sum i^{2}$. And while it may be shown that "For small variations of the current the mean of the squares may be equated to the square of the mean," yet any approximation it may become capable of furnishing is entirely dependent on the hypothesis that the variations are small. But as it is impossible to ensure that they shall be so, any results derived on the assumption that they are must involve an error of indeterminate magnitude.

For these reasons, then, the second method appears much more satisfactory. Up to the present time I have been unable to try it, but shall attempt to do so at a later day.

In conclusion I take this opportunity to express the obligations I feel myself under to Prof. W. F. Magie and Dr. F. A. Waterman, the latter of whom has labored with me in a most kindly way giving hints and suggestions which his work with his own calorimeter has shown him would be advantageous and which have proven so in every instance.

Physical Laboratory, Princeton University. 